\title{
Needleless Electrohydrodynamic Cojetting of Bicompartmental Particles and Fibers from an Extended Fluid Interface
}

\author{
Jacob H. Jordahl, Stacy Ramcharan, Jason V. Gregory, Joerg Lahann*
}

Electrohydrodynamic cojetting can result in fibers (electrospinning) and particles (electrospraying) with complex, bicompartmental architectures. An important consideration for application of bicompartmental particles and fibers is the limited throughput derived from the use of parallel capillaries, which require laminar flow to form a multifluidic interface. Here, a novel synthesis approach that takes advantage of an extended bicompartmental fluid interface formed at the sharp edge of a 2D plate is reported. Upon application of an electrical potential to the plate, several electrified fluid jets form spontaneously. Depending on the processing conditions, either bicompartmental particles or fibers with well-defined architectures are prepared. Importantly, this needleless process yields production rates that are more than 30 times higher than those of conventional needle-based techniques. Fiber properties, such as morphology or size, are independent of the flow rate, indicating that this process is physically self-regulating by adjusting the number of jets ejecting from the extended fluid interface. The needleless preparation of bicompartmental particles and fibers is an important technological breakthrough that can enable further advances ranging from drug delivery and tissue engineering to industrial applications.

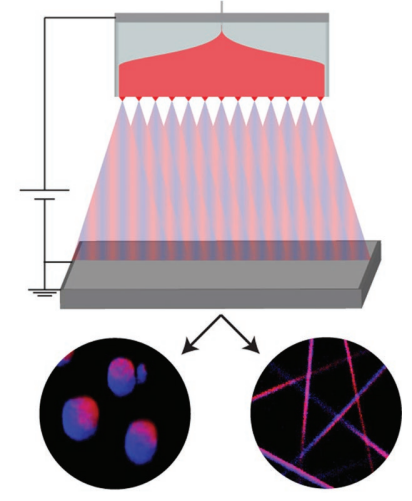

\section{Introduction}

Electrospinning and electrospraying have become technological approaches for creating particles and fibers on small length scales, e.g., dimensions ranging from microns to nanometers in size. ${ }^{[1-3]}$ These techniques

J. H. Jordahl, S. Ramcharan, J. V. Gregory, Prof. J. Lahann Biointerfaces Institute

Department of Chemical Engineering

University of Michigan

2800 Plymouth Rd, Ann Arbor, MI 48109, USA

E-mail: Lahann@umich.edu

Prof. J. Lahann

Department of Biomedical Engineering

Material Science and Engineering, and Macromolecular

Science and Engineering

University of Michigan

2800 Plymouth Rd, Ann Arbor, MI 48109, USA typically involve the transport of polymer solutions through a metal capillary. Upon application of a critical voltage, the droplet at the end of a metal capillary is distorted into a Taylor cone, and a fluid jet is emitted from the cone apex. ${ }^{[2]}$ While the fluid jet traverses toward a grounded electrode, it can either break up into droplets, i.e., electrospraying, ${ }^{[4,5]}$ or is stretched into a fiber, i.e., electrospinning. ${ }^{[6]}$ This technology has been implemented in a variety of applications ranging from chemical sensors, ${ }^{[7,8]}$ filtration membranes, ${ }^{[9,10]}$ and tissue engineering, ${ }^{[10-13]}$ to drug delivery vehicles ${ }^{[5,14]}$ and microencapsulation. ${ }^{[15]}$

More recently, the development of electrohydrodynamic (EHD) cojetting, ${ }^{[16]}$ which imparts multiple chemical functionalities within distinct compartmental domains of both particles and fibers, has shown great promise in creating more complex drug delivery platforms ${ }^{[17-19]}$ as well as patterned targeting domains. ${ }^{[20,21]}$ Additionally, 
multicomponent fibers have shown promise in creating next-generation tissue engineering constructs ${ }^{[22-24]}$ and stimuli-responsive micro- and nanoactuators. ${ }^{[25-27]}$ Scaling this technology to gram scale production has been difficult due to the limitation of one fluid jet per cojetting setup, potentially limiting the commercial viability and widespread adaptation of this technique.

In the case of conventional electrospinning, the limited scalability has been addressed through the development of needleless jetting processes which can produce fibers at rates orders of magnitude greater than what is possible with a single capillary. ${ }^{[28-32]}$ In principle, needleless electrospinning is based on the spontaneous ejection of multiple jets from a fluid reservoir in response to the application of sufficiently high electrical potentials. While this approach has been very successful for conventional electrospinning, it is not directly translatable to electrohydrodynamic cojetting, because the latter requires controlled coflow of two fluids prior to jet formation. Herein, we describe a needleless electrospinning and electrospraying technology, which allows for the fabrication of bicompartmental fibers and particles.

\section{Experimental Section}

\subsection{Materials}

Poly(D,L-lactic-co-glycolic acid) (PLGA, MW 50-75 kg mol ${ }^{-1}$ ), poly(vinyl acetate) (PVAc, MW $113 \mathrm{~kg} \mathrm{~mol}{ }^{-1}$ ), poly[(mphenylenevinylene)-alt-(2,5-dihexyloxy-p-phenylenevinylene)] (PMPDHPV), tetrahydrofuran (THF), chloroform, and $\mathrm{N}, \mathrm{N}-$ dimethylformamide (DMF) were purchased from Sigma Aldrich. Methoxy-polyethylene glycol (PEG)-rhodamine (MW $5 \mathrm{~kg} \mathrm{~mol}^{-1}$ ) was purchased from Creative PEG Works.

\subsection{Device Design}

The microchannel EHD cojetting device was fabricated from 316 stainless steel to an overall size of $76.4 \times 25.5$ (length $\times$ width). A $0.35 \mathrm{~mm}$ microchannel was created on each side of a $0.7 \mathrm{~mm}$ thick plate by placing a raised edge on both sides of the device. The top of the device contained a $2 \mathrm{~mm}$ tall and $3.25 \mathrm{~mm}$ wide ridge, designed for glass slides to mount flush to the device. Fluid inlets were placed on both sides of the metal plate and were centered lengthwise on the ridge of the device. The plate edge at the outlet of the microchannels was sharpened to a point, and grooves were placed approximately every $1 \mathrm{~mm}$ along the edge to aid in fluid flow. Glass slides were cut to size and were mounted onto the device.

\subsection{Particle and Fiber Preparation}

Bicompartmental particles were fabricated using two 6.5 wt\% solutions of PLGA dissolved in a 97:3 ratio of chloroform:DMF. One of the solutions contained $30 \mu \mathrm{g} \mathrm{mL} \mathrm{m}^{-1}$ of PMPDHPV, while the second solution contained $30 \mu \mathrm{g} \mathrm{mL} \mathrm{m}^{-1}$ of $\mathrm{mPEG}$-Rhodamine. Each solution was pumped at flow rates ranging from 1.0 to $13.2 \mathrm{~mL} \mathrm{hr}^{-1}$ (for a total flow rate of 2.0 to $26.4 \mathrm{~mL} \mathrm{hr}^{-1}$ ) into the microchannel device, and a $60 \mathrm{kV}$ electric potential was applied once solution emerged from the microchannels. Flow rates reported represent flow of each individual component. The total flow rate through the device was twice what is reported unless otherwise specified.

Bicompartmental fibers were fabricated using two 35 wt\% solutions of PLGA dissolved in a 1:1 ratio of chloroform:DMF containing similar dye concentrations as the particles. Each solution was pumped into the microchannels at a flow rate up to $13.2 \mathrm{~mL} \mathrm{hr}^{-1}$, for a total of $26.4 \mathrm{~mL} \mathrm{hr}^{-1}$, at an applied electric potential of $75 \mathrm{kV}$.

Bicompartmental fibers were created by flowing PLGA and PVAc polymer solutions on either side of the microchannel EHD cojetting device. A 35 wt\% PLGA solution in a 1:1 THF to DMF solvent system was cospun with a 35 wt\% PVAc solution in a 6:4 chloroform to DMF solvent system. Total flow rates of 2 to $26.4 \mathrm{~mL} \mathrm{hr}^{-1}$ were utilized at a tip to ground distance of $40 \mathrm{~cm}$ and an applied electric potential of $75 \mathrm{kV}$.

Scanning electron microscopy of the particles was performed using an FEI Helios SEM/FIB, while fibers were imaged using an AMRAY 1910 field emission scanning electron microscope. Fiber diameter histograms were determined by ImageJ using the DiameterJ plugin, an approach previously reported in literature. ${ }^{[33]}$ In this process, grayscale images were segmented using an automated thresholding technique. The threshold setting M7 was used for all subsequent analysis.

Fluorescence imaging was conducted with a Nikon A-1 inverted confocal laser scanning microscope (CLSM) using a $60 \times$ oil immersion objective with violet corrected lens, and were processed on Imaris (Version 7.5) software. Particle size distributions were determined by measuring all the particles across $140 \times 240 \mu \mathrm{m}$ quadrants in nine separate fields of view. Particle anisotropy was determined by counting all the particles containing either one or two compartments within nine 3D fields of view within a confocal z-stack. Particles viewed from this perspective could be easily distinguished from one another. Relative compartmental ratios were computed by generating surfaces, which encapsulated the volume of each compartment in Imaris. The volume of each surface on the particle was measured, providing a relative volume ratio for each component.

\section{Results and Discussion}

\subsection{Device Design}

Currently, EHD cojetting requires the use of two capillaries in parallel to create fibers or particles with two or more compartments (Figure 1A). ${ }^{[16]}$ To scale this technology, complex experimental setups involving multiple dual capillary orifices are required. Here, an alternative strategy is explored that relies on the ejection of multiple jets from the edge of an appropriately designed metal plate. Two fluids flow on opposite sides of the plate and combine at the edge to form a stable fluid interface (Figure 1B). It was 

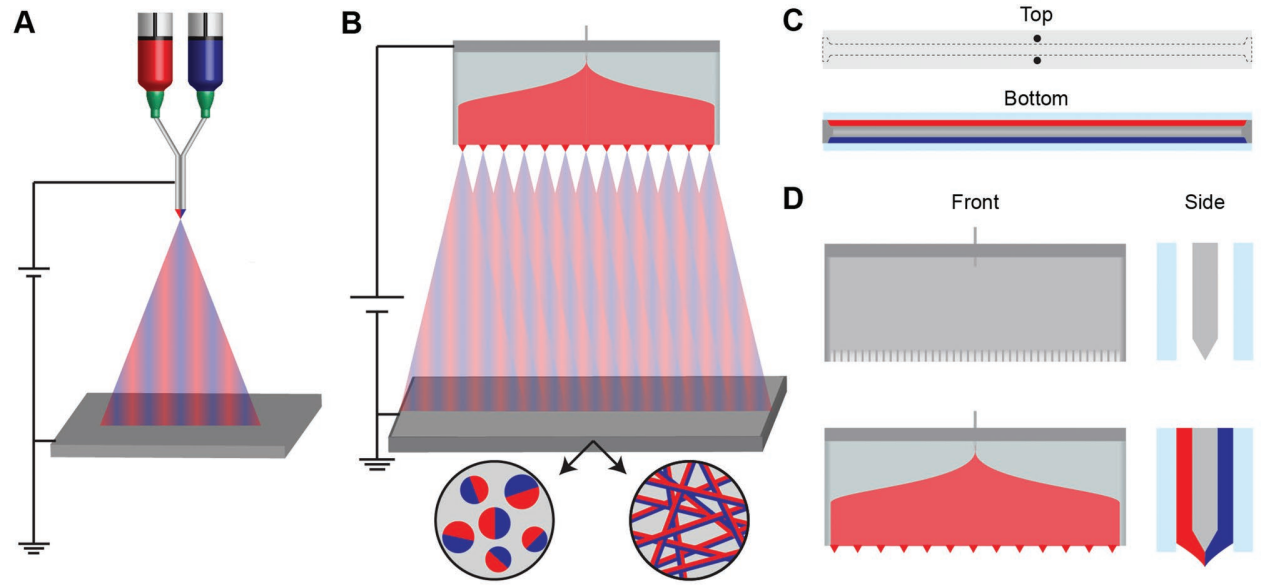

Figure 1. A) A standard EHD cojetting experimental setup includes a single syringe pump, which dispenses two fluids simultaneously through two parallel capillaries. A high voltage is applied to the dual-capillaries, resulting in either electrospinning or electrospraying of bicompartmental fibers or particles respectively. Scaling up this process would require many capillaries in parallel, which is fundamentally impractical. B) A needleless cojetting technique would allow for an increased cone density with high flow rates using a relatively simple design. Operation of this device with results in the deposition of particles or fibers with multiple compartmentalized domains. C) A top and bottom view of the device showing glass slides spaced away from a center plate via raised edges on either side. This creates microchannels for the fluid to flow through. D) The device itself consists of a center plate which is sharpened to an edge to stabilize a biphasic fluid interface with glass walls on either side. Filling the device with polymer solution on either side leads to the formation of an extended fluid interface. Application of electric potential leads to the formation of multiple bicomponent Taylor cones (Movie S1, Supporting Information).

hypothesized that this elongated fluid interface would act similarly to the droplet interface, which is formed by the dual capillaries typically used in EHD cojetting; and that upon application of high voltage spontaneous formation of multiple fluid jets would spontaneously form along the extended fluid interface.

Schematic representations of the top (inlet) and bottom (outlet) of the device show the location of the two microchannels relative to one another, as well as the location of the fluid inlet ports (Figure 1C). Pumping the fluids into the fluid inlet ports of the assembled device allows for the polymer solutions to move on opposite sides of the metal plate. Once the device is filled, a fluid interface is formed at the lower edge. Upon formation of the fluid interface, a high electric potential is applied to the device, forming multiple Taylor cones along the device outlet (Movie S1). The fluid jets formed from the device are then accelerated toward a grounded electrode, where the final particles or fibers are collected and subsequently analyzed (Figure 1D, Movie S2, Supporting Information).

\subsection{Bicompartmental Particle Preparation}

For microparticle fabrication, the needleless cojetting device was infused with two 6.5 wt\% PLGA solutions containing different dyes. Each PLGA solution was infused at flow rates ranging from 2.0 to $26.4 \mathrm{~mL} \mathrm{hr}^{-1}$ to determine the optimum flow rate for bicompartmental particle preparation. For each flow rate, an electric potential of $60 \mathrm{kV}$ was applied to the device. The distance between tip and grounded collector was held constant at $40 \mathrm{~cm}$. It was observed that as the flow rate increased, an increasing number of Taylor cones were formed to maintain a steady state of particle production. The number of Taylor cones increased from three at a $2.0 \mathrm{~mL} \mathrm{hr} r^{-1}$ flow rate, to seven at a flow rate of $26.4 \mathrm{~mL} \mathrm{hr}^{-1}$ along the $7 \mathrm{~cm}$ outlet.

Visualization of the bicompartmental particles was performed using CLSM. Z-stack imaging was utilized to reconstruct the 3D structure of the resultant particles (Figure 2A,B). Analysis of the particles in the z-stack images revealed that at a flow rate of $2.0 \mathrm{~mL} \mathrm{hr}^{-1}, 96.5 \%$ of the particles featured two clearly distinguishable compartments. Within these particles, it was determined that the relative ratio of each compartment was $50.8 \% \pm 4.5 \%(n=150)$. This compares well with the yields described for needle-based cojetting. ${ }^{[34,35]}$ Moreover, statistical analysis of the images obtained by confocal microscopy yielded particle size distributions for the particles prepared with a flow rate of $2.0 \mathrm{~mL} \mathrm{hr}^{-1}$ (Figure 2D). For comparison, electrohydrodynamic cojetting of the same polymer solution using parallel capillaries requires a total flow rate of $0.4 \mathrm{~mL} \mathrm{hr} r^{-1}\left(0.2 \mathrm{~mL} \mathrm{hr}^{-1}\right.$ for each component) to obtain a stable cone-jet. Under otherwise unaltered conditions, the resultant particles had size distributions and particle morphologies that matched the ones observed for needleless jetting seen in Figure 2D (see Figure S1, Supporting Information for comparison).

Not surprisingly, the flow rate had an important influence on particle morphologies: Bicompartmental particles prepared by needleless cojetting ranged from close-to-perfect spherical particles at lower flow rates (Figure 2C) to flattened discs at higher flow rates 


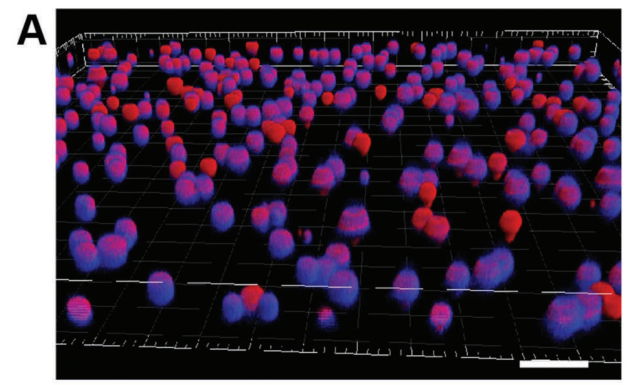

B
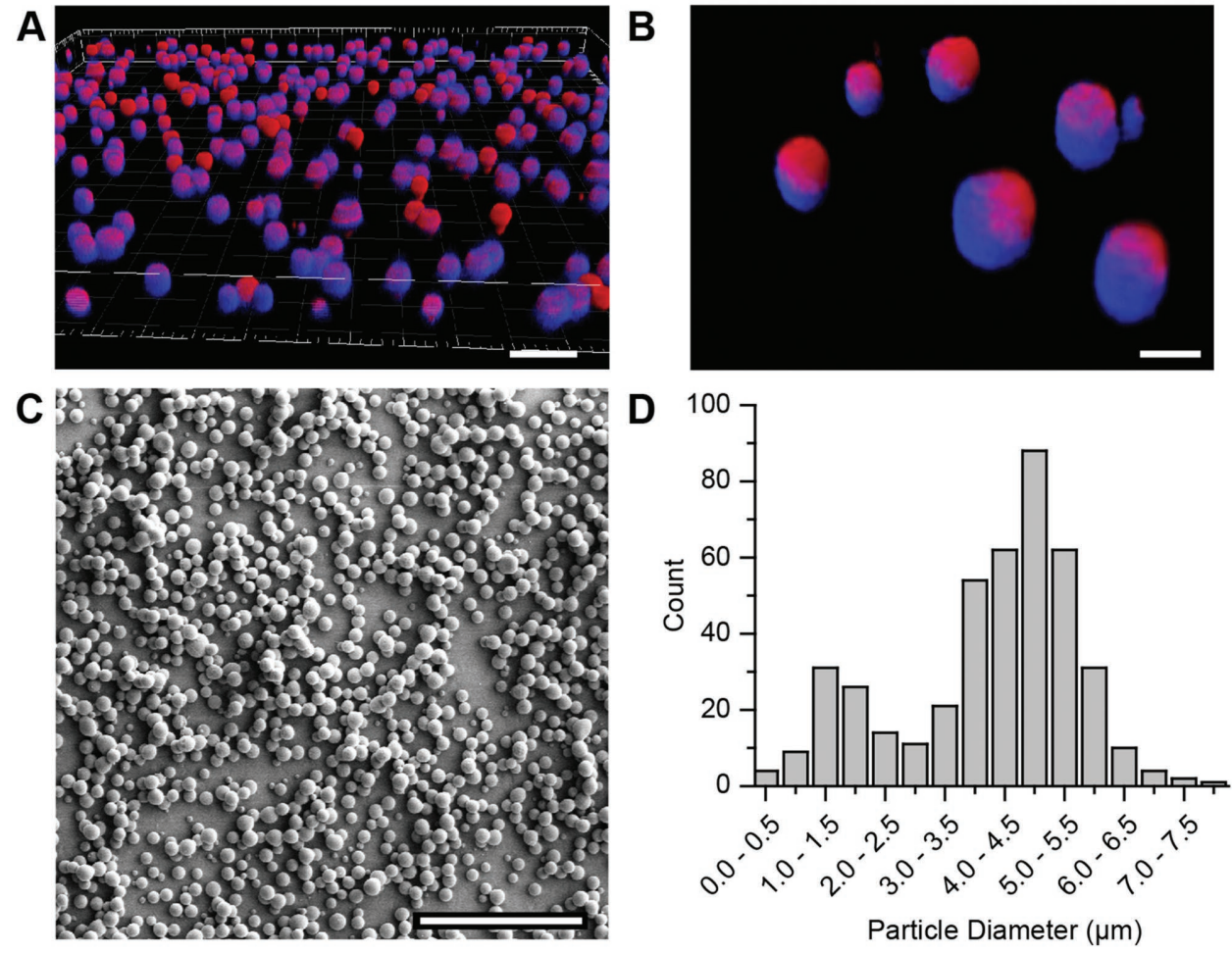

Figure 2. Bicompartmental particles composed of PLGA loaded with different fluorescent dyes fabricated via needleless EHD cojetting device. A) Confocal laser scanning microscopy was used to verify the bicompartmental nature of the particles. B) A zoomed in image of these particles highlights the bicompartmental nature of these particles. C) Scanning electron micrograph of particles fabricated using this device shows particle morphology is consistent with previous EHD cojetting capabilities. D) The particle size distribution as calculated from nine different fields of view via confocal microscopy validates these observations (see Figure S2, Supporting Information). Scale bars indicate (A) $20 \mu \mathrm{m}$, (B) $5 \mu \mathrm{m}$, (C) $50 \mu \mathrm{m}$.

(Figure S2, Supporting Information). A distribution of discs and particles was observed at flow rates as high as $20 \mathrm{~mL} \mathrm{hr}^{-1}$, and discs and red-blood-cell-shaped particles were predominantly observed for flow rates above $26.4 \mathrm{~mL} \mathrm{hr}^{-1}$. We note that disc-shaped particles are generally seen for low concentration polymer solutions pumped at higher flow rates. ${ }^{[34,36]}$ The formation of discs can typically be avoided, when the tip to collector distance is increased. At even higher flow rates, the increased solvent concentration in the atmosphere around the depositing particles leads to a slower evaporation rate, which hinders the solidification of the particle, causing it to flatten into a disc shape. ${ }^{[36]}$ These disc-shaped particles prepared by needleless cojetting were found to still retain a bicompartmental character (Figure S3, Supporting Information).

\subsection{Bicompartmental Fiber Preparation}

Infusion of the microchannel device with two 35 wt\% PLGA solutions, each loaded with a different colored fluorescent dye, resulted in the formation of micron to submicron-sized fibers upon application of a $75 \mathrm{kV}$ electric potential. Again, flow rates from 2.0 to $26.4 \mathrm{~mL} \mathrm{hr}^{-1}$ were examined to determine maximum production rate of bicompartmental fiber mats, and how flow rate affects the overall fiber morphology. For all fiber jetting experiments, the tip to grounded collector distance was maintained at $40 \mathrm{~cm}$ with a constant applied electric potential. Similar increases in Taylor cone numbers with increasing flow rates were observed during fiber electrospinning. However, these cones were more dynamic in nature, and their numbers varied with time. At flow rates of $2.0 \mathrm{~mL} \mathrm{hr}^{-1}$, one to three Taylor cones formed along the outlet of the device, and increasing the flow rate to $26.4 \mathrm{~mL} \mathrm{hr}^{-1}$ produced four to eight Taylor cones.

For all flow rates examined, bicompartmental fibers were obtained. Even at the highest flow rate, the reconstruction of CLSM z-stacks confirmed that the fibers maintained a bicompartmental architecture (Figure 3A,B). CLSM imaging of the cross-sectional view of the fibers further confirms the presence of two distinct compartments (Figure 3B). SEM micrographs reveal fibers with a contiguous, bead-free morphology across all flow rates examined (Figure $3 \mathrm{C}$ ). Under the conditions used for needleless cojetting, fibers had an average diameter of $0.95 \mu \mathrm{m}$ (Figure 3E). Fibers formed at $2 \mathrm{~mL} \mathrm{hr}^{-1}$ had a similar morphology and fiber size distribution as all 

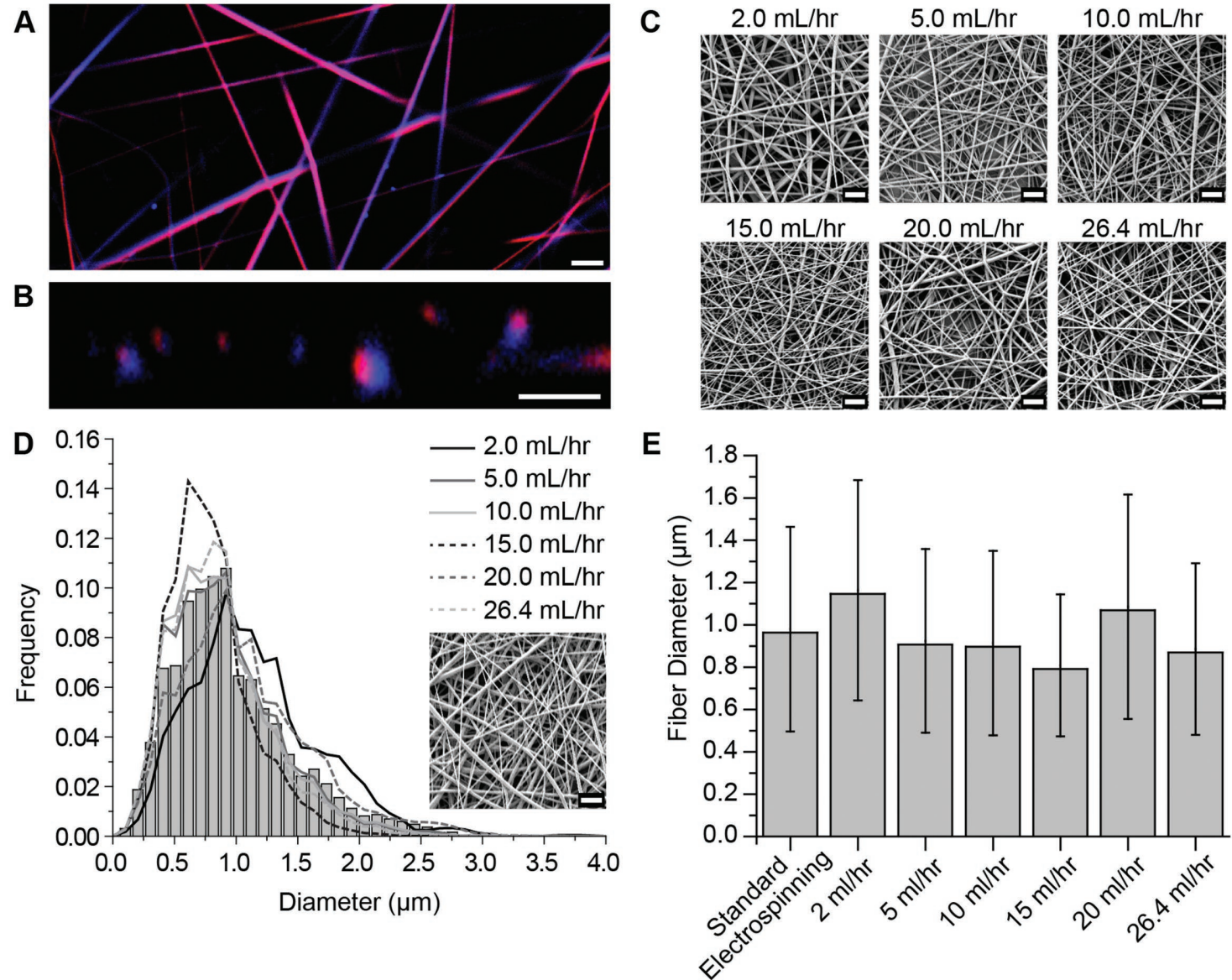

Figure 3. Bicompartmental fibers composed of PLGA compartments loaded with different dyes as fabricated via needleless EHD cojetting device. A) Confocal laser scanning microscopy verifies the bicompartmental fiber architecture is maintained after being processed by the microchannel EHD cojetting device, even at flow rates of $26.4 \mathrm{~mL} \mathrm{hr}^{-1}$. B) A representative cross sectional view of the fibers shown in (A) highlights the two compartments present within the fibers. C) Scanning electron microscopy of fibers produced from 2 to $26.4 \mathrm{~mL} \mathrm{hr}^{-1}$ shows a bead free morphology, which is similar at each respective flow rate. D) A histogram of fiber diameters from fibers spun in a standard side-by-side capillary (SEM shown in inset) is compared to the fiber size distributions of the fibers produced using the microchannel EHD cojetting device at different flow rates, as indicated by the respective lines. E) Average fiber diameter is shown for each of the samples, showing the fiber size is consistent for each condition. Error bars indicate standard deviations. Scale bars indicate $10 \mu \mathrm{m}(\mathrm{A}-\mathrm{D})$.

other fibers, including the highest flow rates examined of $26.4 \mathrm{~mL} \mathrm{hr}^{-1}$. A comparison to fibers fabricated using standard needle-based cojetting using identical PLGA solutions shows a similar fiber size distribution and morphology, demonstrating the stability of this method in fiber production (Figure 3D). This was accomplished despite increasing the overall fiber production rate by over a factor of 30 , from a total flow rate of $0.8 \mathrm{~mL} \mathrm{hr}^{-1}$ in the case of needle-based electrospinning to over $26.4 \mathrm{~mL} \mathrm{hr}^{-1}$ using a needleless device.

Similar results were found when electrospinning of two dissimilar materials was attempted. Cojetting the PLGA solution with PVAc using the needleless device produced fibers with a bicompartmental architecture, with $95.5 \pm 3.4 \%$ of the fibers containing two compartments when electrospun at the highest flow rate of $26.4 \mathrm{~mL} \mathrm{hr}^{-1}$ (Figure 4A,B). The fibers had a consistent bead-free morphology, and a similar fiber size distribution relative to each other and to standard needle-based electrospinning techniques (Figure $4 \mathrm{C}-\mathrm{E}$ ), indicating the system is self-regulating, adding more or less Taylor cones as the flow rate is modulated.

These results demonstrate that fiber production rates of over $9 \mathrm{~g} \mathrm{hr}^{-1}$ (over a 30 fold increase in production rate), well over an order of magnitude higher than typical bicompartmental fiber electrospinning $\left(\approx 0.2 \mathrm{~g} \mathrm{hr}^{-1}\right)$, can be achieved using this novel needleless cojetting technology. These results were consistent for both, fibers loaded with the same base polymer (PLGA) and two different additives (dyes) as well as for fibers comprised of entirely different base polymers (PLGA/PVAc). Further increases in fiber production rate may be feasible, if higher flow rates can be achieved. Increasing the length of the extended edge used to stabilize the fluid interface 
A

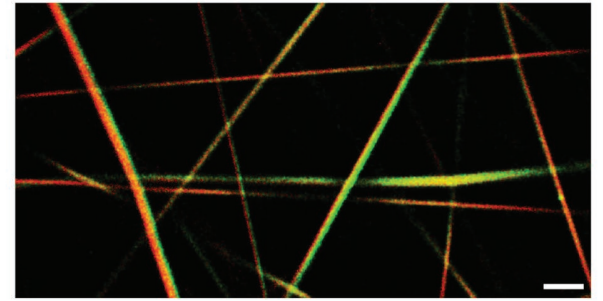

B

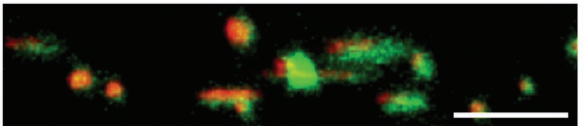

D

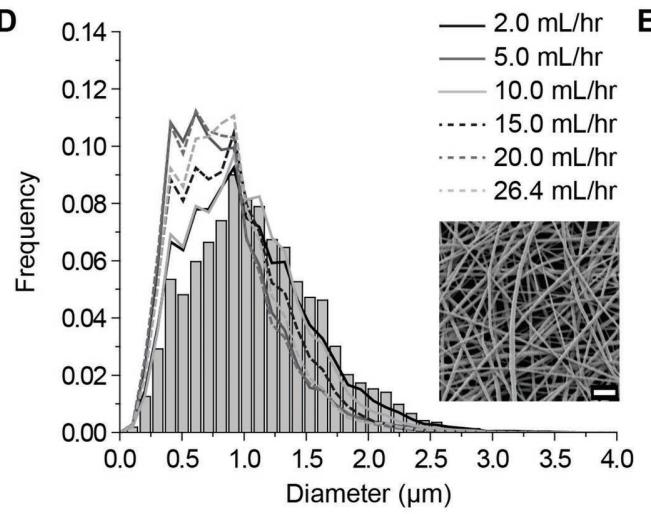

C
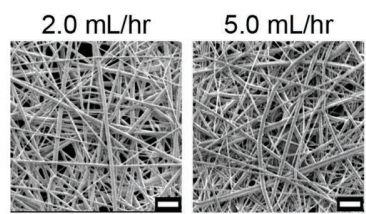

$15.0 \mathrm{~mL} / \mathrm{hr}$

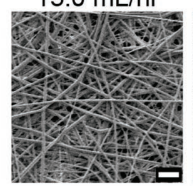

$20.0 \mathrm{~mL} / \mathrm{hr}$
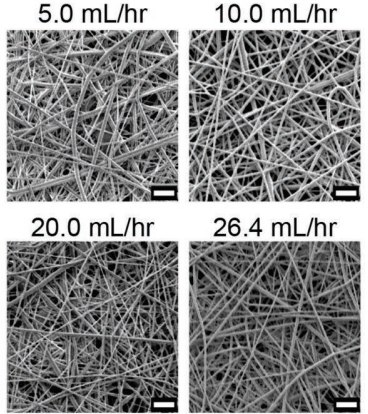

$26.4 \mathrm{~mL} / \mathrm{hr}$

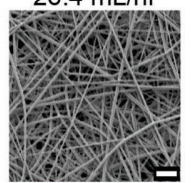

E

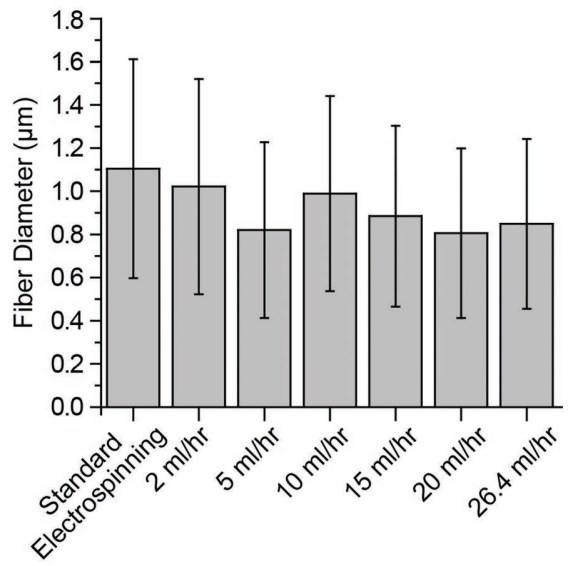

Figure 4. Bicomponent fibers with one compartment containing PLGA (red) and the second containing PVAc (green) were loaded with different dyes and fabricated via needleless EHD cojetting device. A) Confocal laser scanning microscopy verifies the bicompartmental fiber architecture is maintained after being processed by the microchannel EHD cojetting device, even at flow rates of $26.4 \mathrm{~mL} \mathrm{hr}^{-1}$. B) A representative cross sectional view of the fibers shown in (A) highlights the two compartments present within the fibers. C) Scanning electron microscopy of fibers produced from 2 to $26.4 \mathrm{~mL} \mathrm{hr}^{-1}$ shows a bead free morphology, which is similar at each respective flow rate. D) A histogram of fiber diameters from fibers spun in a standard side-by-side capillary (SEM shown in inset) is compared to the fiber size distributions of the fibers produced using the microchannel EHD cojetting device at different flow rates, as indicated by the respective lines. E) Average fiber diameter is shown for each of the samples, showing the fiber size is consistent for each condition. Error bars indicate standard deviations. Scale bars indicate $10 \mu \mathrm{m}(\mathrm{A}-\mathrm{D})$.

would also provide a means of further increasing the rate of production of fibers.

\section{Conclusions}

Bicompartmental particles and fibers, as well as bicomponent fibers containing different base polymers, were successfully fabricated using a newly developed needleless EHD cojetting technique. The device was designed to accommodate two independent fluid flows, which would combine to form a uniform fluid interface at the outlet of a microchannel. Application of a high electric field led to spontaneous formation of several distinct Taylor cones along the fluid interface, resulting in the deposition of bicompartmental fibers and particles. Production rates of fibers were calculated to be on the order of $9 \mathrm{~g} \mathrm{hr}^{-1}$, which is over 30 times higher than standard electrospinning approaches. Spherical particles also had increased production rates, around five times higher than needle-based EHD cojetting. Other bicompartmental particle geometries are also accessible, including discs, which could be fabricated at a rate of nearly $2 \mathrm{~g} \mathrm{hr}^{-1}$. Scaling this technology using larger fluid interfaces and higher flow rates will be examined in future work. This technique provides a potential means for scaling up EHD cojetted particles and fibers for commercial applications.

\section{Supporting Information}

Supporting Information is available from the Wiley Online Library or from the author.

Acknowledgements: The authors would like to acknowledge funding from the Defense Threat Reduction Agency (HDTRA-1-15-1-0045). J.H.J. acknowledges the support of NIH's Microfluidics in Biomedical Sciences Training Program: NIH NIBIB T32 EB005582. S.R. acknowledges the Rackham Graduate School for funding provided by the Rackham Merit Fellowship. 
Received: July 12, 2016; Revised: August 17, 2016; Published online: November 7, 2016; DOI: 10.1002/marc.201600437

Keywords: electrospinning; electrospraying; high-throughput synthesis; Janus fibers; Janus particles; microfluidics

[1] I. K. Kwon, S. Kidoaki, T. Matsuda, Biomaterials 2005, 26, 3929.

[2] D. H. Reneker, I. Chun, Nanotechnology 1996, 7, 216.

[3] A. Greiner, J. H. Wendorff, Angew. Chem. Int. Ed. 2007, 46, 5670.

[4] M. Cloupeau, B. Prunetfoch, J. Aerosol Sci. 1994, 25, 1021.

[5] J. W. Xie, L. K. Lim, Y. Y. Phua, J. S. Hua, C. H. Wang, J. Colloid Interf. Sci. 2006, 302, 103.

[6] D. H. Reneker, A. L. Yarin, H. Fong, S. Koombhongse, J. Appl. Phys. 2000, 87, 4531.

[7] M. S. Kwon, J. H. Jordahl, A. W. Phillips, K. Chung, S. Lee, J. Gierschner, J. Lahann, J. Kim, Chem. Sci. 2016, 7, 2359.

[8] X. Y. Wang, C. Drew, S. H. Lee, K. J. Senecal, J. Kumar, L. A. Sarnuelson, Nano Lett. 2002, 2, 1273.

[9] P. Gibson, H. Schreuder-Gibson, D. Rivin, Colloid Surface A 2001, 187, 469.

[10] Z. M. Huang, Y. Z. Zhang, M. Kotaki, S. Ramakrishna, Compos. Sci. Technol. 2003, 63, 2223.

[11] W. J. Li, C. T. Laurencin, E. J. Caterson, R. S. Tuan, F. K. Ko, J. Biomed. Mater. Res. 2002, 60, 613.

[12] T. J. Sill, H. A. von Recum, Biomaterials 2008, 29, 1989.

[13] H. Yoshimoto, Y. M. Shin, H. Terai, J. P. Vacanti, Biomaterials 2003, 24, 2077.

[14] S. Chakraborty, I. C. Liao, A. Adler, K. W. Leong, Adv. Drug Deliver. Rev. 2009, 61, 1043.

[15] I. G. Loscertales, A. Barrero, I. Guerrero, R. Cortijo, M. Marquez, A. M. Ganan-Calvo, Science 2002, 295, 1695.

[16] K. H. Roh, D. C. Martin, J. Lahann, Nat. Mater. 2005, 4, 759.

[17] S. Rahmani, C. H. Villa, A. F. Dishman, M. E. Grabowski, D. C. Pan, H. Durmaz, A. C. Misra, L. Colon-Melendez, M. J. Solomon, V. R. Muzykantov, J. Lahann, J. Drug Target 2015, 23, 750 .
[18] E. Sokolovskaya, S. Rahmani, A. C. Misra, S. Brase, J. Lahann, ACS Appl. Mater. Inter. 2015, 7, 9744.

[19] S. Rahmani, T. H. Park, A. F. Dishman, J. Lahann, J. Control. Release 2013, 172, 239.

[20] S. Rahmani, A. M. Ross, T. H. Park, H. Durmaz, A. F. Dishman, D. M. Prieskorn, N. Jones, R. A. Altschuler, J. Lahann, Adv. Healthc. Mater. 2016, 5, 94.

[21] S. Rahmani, S. Saha, H. Durmaz, A. Donini, A. C. Misra, J. Yoon, J. Lahann, Angew. Chem. Int. Ed. 2014, 53, 2332.

[22] S. Bhaskar, K. H. Roh, X. W. Jiang, G. L. Baker, J. Lahann, Macromol. Rapid Comm. 2008, 29, 1973.

[23] S. Mandal, S. Bhaskar, J. Lahann, Macromol. Rapid Comm. 2009, 30, 1638

[24] J. Yoon, T. W. Eyster, A. C. Misra, J. Lahann, Adv. Mater. 2015, $27,4509$.

[25] J. Lee, T. H. Park, K. J. Lee, J. Lahann, Macromol. Rapid Comm. 2016, 37, 73.

[26] S. Saha, D. Copic, S. Bhaskar, N. Clay, A. Donini, A. J. Hart, J. Lahann, Angew. Chem. Int. Ed. 2012, 51, 660.

[27] K. J. Lee, J. Yoon, S. Rahmani, S. Hwang, S. Bhaskar, S. Mitragotri, J. Lahann, Proc. Natl. Acad. Sci. USA 2012, 109, 16057.

[28] A. L. Yarin, E. Zussman, Polymer 2004, 45, 2977.

[29] B. A. Lu, Y. J. Wang, Y. X. Liu, H. G. Duan, J. Y. Zhou, Z. X. Zhang, Y. O. Wang, X. D. Li, W. Wang, W. Lan, E. O. Xie, Small 2010, 6, 1612

[30] N. M. Thoppey, J. R. Bochinski, L. I. Clarke, R. E. Gorga, Polymer 2010, 51, 4928.

[31] X. Wang, H. T. Niu, T. Lin, X. G. Wang, Polym. Eng. Sci. 2009, 49, 1582.

[32] K. M. Forward, G. C. Rutledge, Chem. Eng. J. 2012, 183, 492.

[33] N. A. Hotaling, K. Bharti, H. Kriel, C. G. Simon, Biomaterials 2015, 61, 327.

[34] S. Bhaskar, K. M. Pollock, M. Yoshida, J. Lahann, Small 2010, 6,404 .

[35] M. Yoshida, K. H. Roh, S. Mandal, S. Bhaskar, D. W. Lim, H. Nandivada, X. P. Deng, J. Lahann, Adv. Mater. 2009, 21, 4920.

[36] N. Bock, M. A. Woodruff, D. W. Hutmacher, T. R. Dargaville, Polymers-Basel 2011, 3, 131. 